\title{
PRIMITIVE POLYNOMIALS OVER FINITE FIELDS
}

\author{
TOM HANSEN AND GARY L. MULLEN
}

\begin{abstract}
In this note we extend the range of previously published tables of primitive polynomials over finite fields. For each $p^{n}<10^{50}$ with $p \leq 97$ we provide a primitive polynomial of degree $n$ over $F_{p}$. Moreover, each polynomial has the minimal number of nonzero coefficients among all primitives of degree $n$ over $F_{p}$.
\end{abstract}

\section{INTRODUCTION}

Let $F_{q}$ denote the finite field of order $q=p^{n}$, where $p$ is prime and $n \geq$ 1. The multiplicative group $F_{q}^{*}$ of nonzero elements of $F_{q}$ is cyclic and a generator of $F_{q}^{*}$ is called a primitive element. Moreover, a monic irreducible polynomial whose roots are primitive elements is called a primitive polynomial. It is well known that the field $F_{q}$ can be constructed as $F_{p}[x] /(f(x))$, where $f(x)$ is an irreducible polynomial of degree $n$ over $F_{p}$ and, in addition, if $f(x)$ is primitive, then $F_{q}^{*}$ is generated multiplicatively by any root of $f(x)$. With the recent availability of faster machines there is a need to significantly extend the range of published tables of primitive polynomials so as to be able to implement the arithmetic of larger fields for various applications in a variety of areas. In this note we exhibit for each prime power $p^{n}<10^{50}$ with $p \leq 97$ a primitive polynomial of degree $n$ over $F_{p}$. Moreover, for each such $p$ and $n$ we have listed a primitive of degree $n$ over $F_{p}$ with the minimal number of nonzero coefficients among all primitives of degree $n$ over $F_{p}$. In addition to the tables presented in $\S 4$ we propose in $\S 5$ two conjectures concerning the distribution of primitive and irreducible polynomials over finite fields.

\section{Published tables}

Table F of Lidl and Niederreiter [9], which is taken from Alanen and Knuth [1], lists one primitive of degree $n$ over $F_{p}$ for $p^{n}<10^{9}$ with $p \leq 47$. Sugimoto [14] extended this for the same primes $p$ to the range $p^{n}<10^{19}$. Because of applications in a variety of areas, including information theory, tables with larger ranges are available for $p=2$. In particular, Watson [15] gives for $n \leq 100$ one primitive of degree $n$ over $F_{2}$, and Stahnke [13] lists for each $n \leq 168$ a primitive with a minimum number of nonzero coefficients.

Received by the editor February 7, 1991 and, in revised form, October 8, 1991.

1991 Mathematics Subject Classification. Primary 11 T06.

Key words and phrases. Finite field, primitive polynomial.

The authors would like to thank the NSA for partial support under the second author's grant agreement \#MDA904-87-H-2023. 
Zierler and Brillhart $[17,18]$ greatly extended this work by listing all primitive trinomials of degree $n$ over $F_{2}$ with $n \leq 1000$. The tables of [17,18] also give all irreducible trinomials of degree $n \leq 1000$ and their orders. Green and Taylor [8] list one primitive over $F_{q}$ of degree $n$ with $q=4, n \leq 11 ; q=$ $8, n \leq 7 ; q=9, n \leq 7 ;$ and $q=16, n \leq 5$. Beard and West [3] study special types of primitive polynomials over $F_{q}$, and Peterson and Weldon [12] give all irreducibles over $F_{2}$ with $n \leq 16$. For $17 \leq n \leq 34$ they give a primitive with a minimum number of nonzero coefficients and an irreducible belonging to each possible order.

\section{Primitive polynomials}

Given a monic polynomial of degree $n$ over $F_{q}$, the following provides an algorithm to test for primitivity, see Lidl and Niederreiter [9, Theorem 3.18].

Theorem 1. The monic polynomial $f \in F_{q}[x]$ of degree $n \geq 1$ is a primitive polynomial over $F_{q}$ if and only if $(-1)^{n} f(0)$ is a primitive element of $F_{q}$ and the least positive integer $r$ for which $x^{r}$ is congruent $\bmod f(x)$ to some element of $F_{q}$ is $r=\left(q^{n}-1\right) /(q-1)$. If $f(x)$ is primitive over $F_{q}$, then $x^{r} \equiv(-1)^{n} f(0)$ $(\bmod f(x))$.

The following algorithm, which is a simplified version of Theorem 1, was implemented on a SUN 460 workstation to test a polynomial $f(x)$ of degree $n$ over $F_{p}$ for primitivity. As a precomputation, for a given $p$ and $n$, the prime factorization of $p^{n}-1$ was obtained using the computer programs Mathematica [16], PARI [2], a General Factorization and Primality Testing Program [4], and the tables from the Cunningham Project [5].

Only those $f(x)$ for which $(-1)^{n} f(0)$ is a primitive element in $F_{p}$ need be considered. First, $f(\theta)$ is calculated for each $\theta \in F_{p}^{*}$ to eliminate those $f$ 's with linear factors. Then the rank of the Berlekamp matrix is calculated to eliminate reducible polynomials for which the rank is of course less than $n-1$, see $[9, \S 4.1]$.

The residue of $x^{\left(p^{n}-1\right) /(p-1)} \quad(\bmod f(x))$ is calculated, and if $x^{\left(p^{n}-1\right) /(p-1)} \not \equiv$ $(-1)^{n} f(0)(\bmod f(x))$, then $f(x)$ is not primitive. If $x^{\left(p^{n}-1\right) /(p-1)} \equiv(-1)^{n} f(0)$ $(\bmod f(x))$, we proceed as follows. For each prime factor $s$ of $\left(p^{n}-1\right) /(p-1)$ such that $s$ does not divide $p-1$, the residue of $x^{\left(p^{n}-1\right) /((p-1) s)}(\bmod f(x))$ is calculated. If, for one such $s$ we have $x^{\left(p^{n}-1\right) /((p-1) s)} \equiv b(\bmod f(x))$ with $b \in F_{q}$, then $f(x)$ is not primitive. If for all such $s, x^{\left(p^{n}-1\right) /((p-1) s)} \not \equiv b$ $(\bmod f(x))$ with $b \in F_{q}$, then $f(x)$ is a primitive polynomial of degree $n$ over $F_{p}$.

\section{TABLES}

In the Supplement section at the end of this issue we provide tables of the primitive polynomials obtained from the calculations described in $\S 3$. For each $p^{n}<10^{50}$ with $p \leq 97$, we provide a primitive polynomial of degree $n$ over $F_{p}$. Moreover, each polynomial has the minimal number of nonzero coefficients (minimal Hamming weight) among all primitives of degree $n$ over $F_{p}$.

In our search procedure, for a given $p$ and $n$, we first tried to locate a primitive trinomial of degree $n$ over $F_{p}$. Failing this, a search was conducted among polynomials of Hamming weight four, then five, etc. Among those polynomials 
of a given weight, say among trinomials for example, polynomials were tested for primitivity in the following order. Consider $f(x)=x^{n}+\sum_{i=0}^{n-1} a_{i} x^{i}$ of degree $n$ over $F_{p}$. Let $N_{f}=p^{n}+\sum_{i=0}^{n-1} a_{i} p^{i}$ be the corresponding number in base $p$. Thus, among the trinomials of degree $n$ over $F_{p}, f(x)$ was tested for primitivity before $g(x)$ if $N_{f}<N_{g}$. Subject to this ordering, the first primitive polynomial obtained is listed in the table.

Each polynomial has minimal Hamming weight among all primitives of degree $n$ over $F_{p}$. Primitive 5-nomials were of minimal weight for some values of $n$ in the $p=2$ case, and for $p=3$ with $n=48,72,96$. In all other cases we have a primitive of degree $n$ with weight at most 4 .

In the tables only the nonzero terms are represented, so that for example over $F_{7}$, the polynomial $x^{14}+2 x^{5}+3$ is represented as $14: 1,5: 2,0: 3$. Copies of the tables and/or programs, either in electronic or hardcopy form, are available upon request from the authors.

\section{TWO CONJECTURES}

Before closing, we raise two conjectures concerning the distribution of primitive and irreducible polynomials over finite fields. We also provide some evidence for each of the conjectures.

Conjecture A. Let $a \in F_{q}$, let $n \geq 2$ and fix $0 \leq j<n$. Then there exists $a$ primitive polynomial $f(x)=x^{n}+\sum_{k=0}^{n-1} a_{k} x^{k}$ of degree $n$ over $F_{q}$ with $a_{j}=a$ except when

(A1) $q$ arbitrary, $j=0$, and $a \neq(-1)^{n} \alpha$, where $\alpha \in F_{q}$ is a primitive element;

(A2) $q$ arbitrary, $n=2, j=1$, and $a=0$;

(A3) $q=4, n=3, j=2$, and $a=0$;

(A4) $q=4, n=3, j=1$, and $a=0$;

(A5) $q=2, n=4, j=2$, and $a=1$.

Theorem 1 implies that the constant term of a primitive polynomial must be of the form $(-1)^{n} \alpha$ with $\alpha$ primitive in $F_{q}$, and hence (A1) is a necessary exception. Clearly, $x^{2}+a$ cannot be primitive over $F_{q}$, and so we have (A2). From [8, Table 1] we deduce the exceptions (A3) and (A4). Exceptions (A3) and (A4) will also be excluded as a result of Theorem 2 below. Exception (A5) arises from Table $\mathrm{F}$ of [9].

Conjecture A states that with the five necessary exceptions, there exists a primitive polynomial of degree $n$ over $F_{q}$ with the coefficient of any fixed power of $x$ prescribed in advance.

For irreducible polynomials we propose:

Conjecture B. Let $a \in F_{q}$, let $n \geq 2$ and fix $0 \leq j<n$. Then there exists an irreducible polynomial $f(x)=x^{n}+\sum_{k=0}^{n-1} a_{k} x^{k}$ over $F_{q}$ with $a_{j}=a$ except when

(B1) $q$ arbitrary and $j=a=0$;

(B2) $q=2^{m}, n=2, j=1$, and $a=0$. 
Clearly, (B1) must be an exception, for otherwise $f(x)$ is divisible by $x$. As for (B2), in characteristic two, every element of $F_{q}$ is a square, and so $x^{2}+a=(x+b)^{2}$ is reducible. Conditions (A3) and (A4) for primitivity may now be removed because such irreducibles exist by [8, Table 1]. Similarly, (A5) may be removed because of Table $F$ of [9].

As evidence for Conjectures A and B we first note that Table F of [9] supports both conjectures for small $p$ and $n$, and Tables 1-4 of [8] support the conjectures for small $n$ and nonprime $q$. The chief theoretical result in this direction is the following result of Cohen [7, Theorem 1]. We remind the reader that, if $n \geq 2$, the trace function is defined from $F_{q^{n}}$ to $F_{q}$ by $\operatorname{TR}(\gamma)=\gamma+\gamma^{q}+\gamma^{q^{2}}+\cdots+\gamma^{q^{n-1}}$.

Theorem 2. Let $n \geq 2$ and let $a \in F_{q}$ with $a \neq 0$ if $n=2$ or if $n=3$ and $q=4$. Then there exists a primitive polynomial of degree $n$ over $F_{q}$ with trace $a$.

Cohen [7] proved that $F_{q^{n}}$ contains a primitive element $\gamma$ with $\operatorname{TR}(\gamma)=a$ over $F_{q}$, where the trace of $\gamma$ is of course the negative of the coefficient of $x^{n-1}$ in the minimal polynomial of $\gamma$ over $F_{q}$. Cohen's theorem explains the exceptions (A2) and (A3) and indirectly (A4), since if $f(x)$ is primitive, so is the reciprocal polynomial $f^{*}(x)$ of $f(x)$, namely, $f^{*}(x)=x^{n} f(1 / x)$, which accounts for (A4). His result proves that among the primitives of degree $n$, the coefficients of $x^{n-1}$ satisfy Conjecture $\mathrm{A}$, and since every primitive is irreducible, Conjecture B as well.

We now show that the constant terms of the primitive and irreducible polynomials satisfy Conjectures $\mathrm{A}$ and $\mathrm{B}$. For Conjecture A, let $\alpha$ be a primitive element in $F_{q^{n}}$ and let $f_{\alpha}(x)$ be the minimal polynomial of $\alpha$ over $F_{q}$ with constant term $(-1)^{n} a$, where by Theorem $1, a$ is a primitive element in $F_{q}$ and moreover, $a=\alpha^{\left(q^{n}-1\right) /(q-1)}$. Let $b$ be a primitive element in $F_{q}$ and let $b=a^{l}$ with $1 \leq l \leq q-2$. Choose $k$ so that $\left(k, q^{n}-1\right)=1$ and $k \equiv l$ $(\bmod q-1)$. Then $\alpha^{k}$ is primitive in $F_{q^{n}}$ and hence the minimal polynomial $g(x)$ of $\alpha^{k}$ is a monic primitive of degree $n$ over $F_{q}$ and, moreover, the constant term of $g(x)$ is $(-1)^{n} \alpha^{k\left(q^{n}-1\right) /(q-1)}=(-1)^{n} a^{k}=(-1)^{n} a^{l}=(-1)^{n} b$.

For Conjecture $\mathrm{B}$, let $c \neq 0 \in F_{q}$, so $c=a^{m}$ with $0 \leq m \leq q-2$. The element $\alpha^{m}$ cannot be in any proper subfield of $F_{q^{n}}$, for otherwise $\alpha^{m\left(q^{t}-1\right)}=1$ with $t \mid n$, a contradiction, since $\alpha$ has order $q^{n}-1$. Hence, the minimal polynomial $h(x)$ of $\alpha^{m}$ is a monic irreducible of degree $n$ over $F_{q}$ with constant term $(-1)^{n} \alpha^{m\left(q^{n}-1\right) /(q-1)}=(-1)^{n} c$. Thus, the constant terms indeed satisfy Conjectures A and B.

From the above discussion we see that Conjectures A and B hold for polynomials of degree two. We also note that if $f(x)$ is irreducible, so is $f(x+e)$ for $e \in F_{q}$, and if a primitive (irreducible) polynomial $f(x)$ has 0 coefficient of $x^{n-k}$ for $1 \leq k<n$, then the primitive (irreducible) polynomial $(1 / f(0)) f^{*}(x)$ has 0 coefficient of $x^{k}$, where $f^{*}(x)$ is the reciprocal of $f(x)$. Finally, if $f(x)$ is irreducible of degree $n$ over $F_{q}$, then $f^{Q}(x)=x^{n} f(x+1 / x)$ is irreducible if and only if $x^{2}-\beta x+1$ is irreducible over $F_{q^{n}}$, where $\beta$ is any root of $f(x)$, see Meyn [10, Lemma 5], also Niederreiter [11] and Cohen [6] for related results. These simple transformations can of course be repeatedly applied to obtain further evidence for the conjectures. 
If Conjectures $\mathrm{A}$ and $\mathrm{B}$ are true, then the primitive and irreducible polynomials of fixed degree over $F_{q}$ are in a limited way rather uniformly distributed over $F_{q}$.

\section{ACKNOWLEDGMENT}

We would like to thank C. Batut, D. Bernardi, H. Cohen, and M. Olivier for providing a copy of PARI and D. M. Bressoud for providing a copy of A General Factorization and Primality Testing Program that were extremely helpful in our calculations. Thanks are also due J. V. Brawley and W.-S. Chou for helpful discussions.

\section{BIBLIOGRAPHY}

1. J. D. Alanen and D. E. Knuth, Tables of finite fields, Sankhyā Ser. A 26 (1964), 305-328.

2. C. Batut, D. Bernardi, H. Cohen, and M. Olivier, PARI, Version 1.32, 1989, 1990.

3. J. T. B. Beard, Jr. and K. I. West, Some primitive polynomials of the third kind, Math. Comp. 28 (1974), 1166-1167.

4. D. M. Bressoud, $A$ general factorization and primality testing program, The Pennsylvania State University, 1988.

5. J. Brillhart, D. H. Lehmer, J. L. Selfridge, B. Tuckerman, and S. S. Wagstaff, Jr., Factorizations of $b^{n} \pm 1, b=2,3,5,6,7,10,11,12$ up to high powers, Contemp. Math., Vol. 22, Amer. Math. Soc., Providence, R. I., 1983.

6. S. D. Cohen, On irreducible polynomials of certain types in finite fields, Proc. Cambridge Philos. Soc. 66 (1969), 335-344.

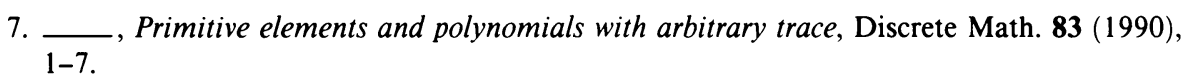

8. D. H. Green and I. S. Taylor, Irreducible polynomials over composite Galois fields and their applications in coding techniques, Proc. IEE 121 (1974), 935-939.

9. R. Lidl and H. Niederreiter, Finite fields, Encyclopedia Math. Appl., Vol. 20, AddisonWesley, Reading, Mass., 1983 (Now distributed by Cambridge Univ. Press).

10. H. Meyn, On the construction of irreducible self-reciprocal polynomials over finite fields, Applicable Algebra in Eng., Comm. and Comp. 1 (1990), 43-53.

11. H. Niederreiter, An enumeration formula for certain irreducible polynomials with an application to the construction of irreducible polynomials over the binary field, Applicable Algebra in Eng., Comm. and Comp. 1 (1990), 119-124.

12. W. W. Peterson and E. J. Weldon, Jr., Error-correcting codes, 2nd ed., M.I.T. Press, Cambridge Mass., 1972.

13. W. Stahnke, Primitive binary polynomials, Math. Comp. 27 (1973), 977-980.

14. E. Sugimoto, A short note on new indexing polynomials of finite fields, Inform. and Control 41 (1979), 243-246.

15. E. J. Watson, Primitive polynomials $(\bmod 2)$, Math. Comp. 16 (1962), 368-369.

16. S. Wolfram, Mathematica (sun 3.68881) 1.2, 1988, 1989.

17. N. Zierler and J. Brillhart, On primitive trinomials $(\bmod 2)$, Inform. and Control 13 (1968), 541-554.

18. __ On primitive trinomials $(\bmod 2)$, II, Inform. and Control $14(1969), 566-569$.

Department of Mathematics, The Pennsylvania State University, University Park, Pennsylvania 16802

E-mail address, G. L. Mullen: mullen@math.psu.edu

E-mail address, T. Hansen: pho3@math.psu.edu 


\title{
Supplement to
}

\section{PRIMITIVE POLYNOMIALS OVER FINITE FIELDS}

\author{
TOM HANSEN AND GARY L. MULLEN
}

In the tables which follow, we have given for each prime power $p^{n}<10^{50}$ with $p \leq 97$, a primitive polynomial of degree $n$ over $F_{p}$. Moreover, each polynomial has minimal weight, i.e., the minimal number of nonzero coefficients, among all primitives of that degree over $F_{p}$.

Only the nonzero terms are represented, so that for example over $F_{7}$, the polynomial $x^{14}+2 x^{5}+3$ is represented as $14: 1,5: 2,0: 3$. Copies of the tables and/or programs, either in electronic or hardcopy form, are available upon request from the authors.

For further details, we refer to the paper of the same title by the authors in this issue of Mathematics of Computation.

$\begin{array}{ll}\text { p=2 } & 54: 1,8: 1,6: 1,3: 1,0: 1 \\ 2: 1,1: 1,0: 1 & 55: 1,24: 1,0: 1 \\ 3: 1,1: 1,0: 1 & 56: 1,7: 1,4: 1,2: 1,0: 1 \\ 4: 1,1: 1,0: 1 & 57: 1,7: 1,0: 1 \\ 5: 1,2: 1,0: 1 & 58: 1,19: 1,0: 1 \\ 6: 1,1: 1,0: 1 & 59: 1,7: 1,4: 1,2: 1,0: 1 \\ 7: 1,1: 1,0: 1 & 60: 1,1: 1,0: 1 \\ 8: 1,4: 1,3: 1,2: 1,0: 1 & 61: 1,5: 1,2: 1,1: 1,0: 1 \\ 9: 1,4: 1,0: 1 & 62: 1,6: 1,5: 1,3: 1,0: 1 \\ 10: 1,3: 1,0: 1 & 63: 1,1: 1,0: 1 \\ 11: 1,2: 1,0: 1 & 64: 1,4: 1,3: 1,1: 1,0: 1 \\ 12: 1,6: 1,4: 1,1: 1,0: 1 & 65: 1,18: 1,0: 1 \\ 13: 1,4: 1,3: 1,1: 1,0: 1 & 66: 1,9: 1,8: 1,6: 1,0: 1 \\ 14: 1,5: 1,3: 1,1: 1,0: 1 & 67: 1,5: 1,2: 1,1: 1,0: 1 \\ 15: 1,1: 1,0: 1 & 68: 1,9: 1,0: 1 \\ 16: 1,5: 1,3: 1,2: 1,0: 1 & 69: 1,6: 1,5: 1,2: 1,0: 1 \\ 17: 1,3: 1,0: 1 & 70: 1,5: 1,3: 1,1: 1,0: 1 \\ 18: 1,7: 1,0: 1 & 71: 1,6: 1,0: 1 \\ 19: 1,5: 1,2: 1,1: 1,0: 1 & 72: 1,10: 1,9: 1,3: 1,0: 1 \\ 20: 1,3: 1,0: 1 & 73: 1,25: 1,0: 1 \\ 21: 1,2: 1,0: 1 & 74: 1,7: 1,4: 1,3: 1,0: 1 \\ 22: 1,1: 1,0: 1 & 75: 1,6: 1,3: 1,1: 1,0: 1 \\ 23: 1,5: 1,0: 1 & 70: 1,5: 1,4: 1,2: 1,0: 1 \\ 24: 1,4: 1,3: 1,1: 1,0: 1 & 77: 1,6: 1,5: 1,2: 1,0: 1 \\ 25: 1,3: 1,0: 1 & 78: 1,7: 1,2: 1,1: 1,0: 1 \\ 26: 1,6: 1,2: 1,1: 1,0: 1 & 79: 1,9: 1,0: 1 \\ 27: 1,5: 1,2: 1,1: 1,0: 1 & 80: 1,9: 1,4: 1,2: 1,0: 1 \\ 28: 1,3: 1,0: 1 & 81: 1,4: 1,0: 1 \\ 29: 1,2: 1,0: 1 & 82: 1,9: 1,6: 1,4: 1,0: 1 \\ 30: 1,6: 1,4: 1,1: 1,0: 1 & 83: 1,7: 1,4: 1,2: 1,0: 1 \\ 31: 1,3: 1,0: 1 & 84: 1,13: 1,0: 1 \\ 32: 1,7: 1,6: 1,2: 1,0: 1 & 85: 1,8: 1,2: 1,1: 1,0: 1 \\ 33: 1,13: 1,0: 1 & 86: 1,6: 1,5: 1,2: 1,0: 1 \\ 34: 1,8: 1,4: 1,3: 1,0: 1 & 87: 1,13: 1,0: 1 \\ 35: 1,2: 1,0: 1 & 88: 1,11: 1,9: 1,8: 1,0: 1 \\ 36: 1,11: 1,0: 1 & 89: 1,38: 1,0: 1 \\ 37: 1,6: 1,4: 1,1: 1,0: 1 & 90: 1,5: 1,3: 1,2: 1,0: 1 \\ 38: 1,6: 1,5: 1,1: 1,0: 1 & 91: 1,8: 1,5: 1,1: 1,0: 1 \\ 39: 1,4: 1,0: 1 & 92: 1,6: 1,5: 1,2: 1,0: 1 \\ 40: 1,5: 1,4: 1,3: 1,0: 1 & 93: 1,2: 1,0: 1 \\ 41: 1,3: 1,0: 1 & 94: 1,21: 1,0: 1 \\ 42: 1,7: 1,4: 1,3: 1,0: 1 & 95: 1,11: 1,0: 1 \\ 43: 1,6: 1,4: 1,3: 1,0: 1 & 96: 1,10: 1,9: 1,6: 1,0: 1 \\ 44: 1,6: 1,5: 1,2: 1,0: 1 & 97: 1,6: 1,0: 1 \\ 45: 1,4: 1,3: 1,1: 1,0: 1 & 98: 1,11: 1,0: 1 \\ 46: 1,8: 1,7: 1,6: 1,0: 1 & 99: 1,7: 1,5: 1,4: 1,0: 1 \\ 47: 1,5: 1,0: 1 & 100: 1,37: 1,0: 1 \\ 48: 1,9: 1,7: 1,4: 1,0: 1 & 101: 1,7: 1,6: 1,1: 1,0: 1 \\ 49: 1,9: 1,0: 1 & 102: 1,0: 1,5: 1,3: 1,0: 1 \\ 50: 1,4: 1,3: 1,2: 1,0: 1 & 103: 1,9: 1,0: 1 \\ 51: 1,6: 1,3: 1,1: 1,0: 1 & 104: 1,11: 1,10: 1,1: 1,0: 1 \\ 52: 1,3: 1,0: 1 & 105: 1,16: 1,0: 1 \\ 53: 1,6: 1,2: 1,1: 1,0: 1 & 106: 1,15: 1,0: 1 \\ & \end{array}$

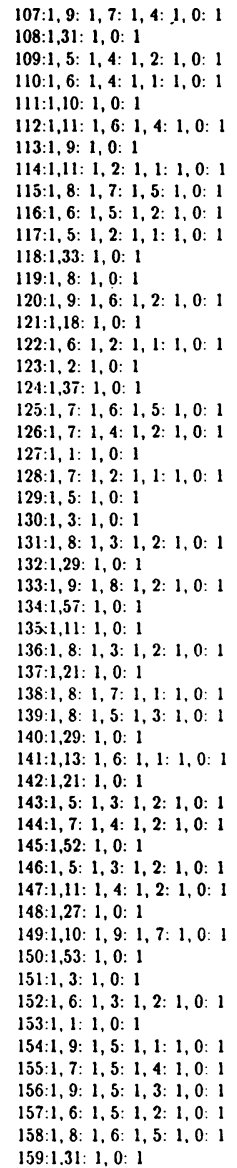

(C) 1992 American Mathematical Society $0025-5718 / 92 \$ 1: 00+\$ .25$ per page 


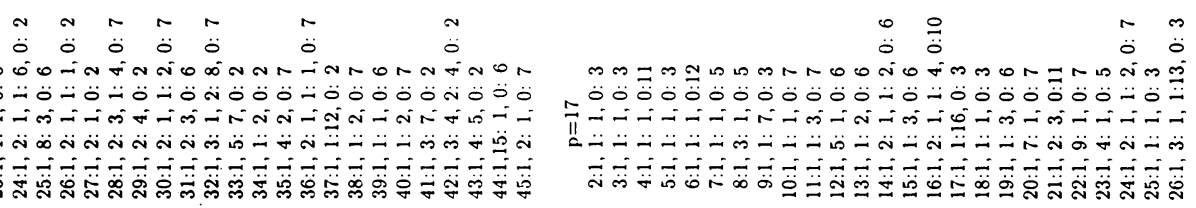

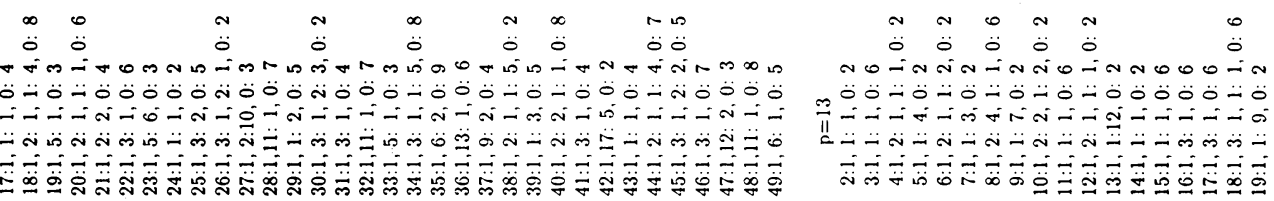

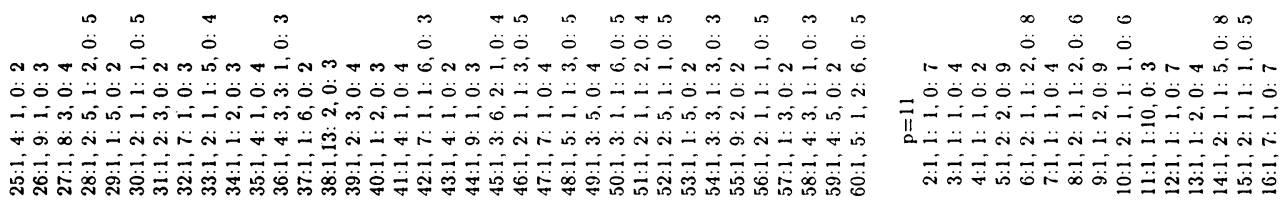

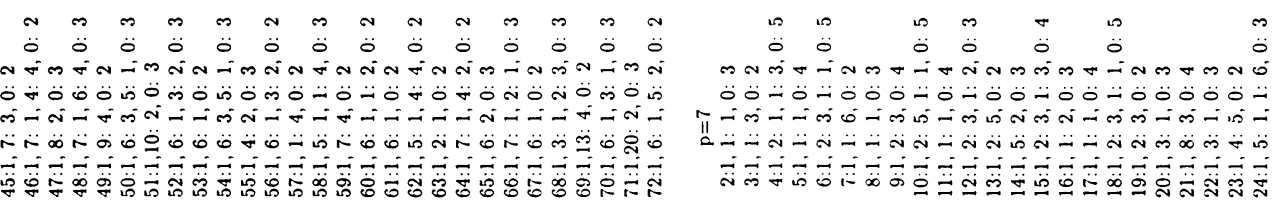

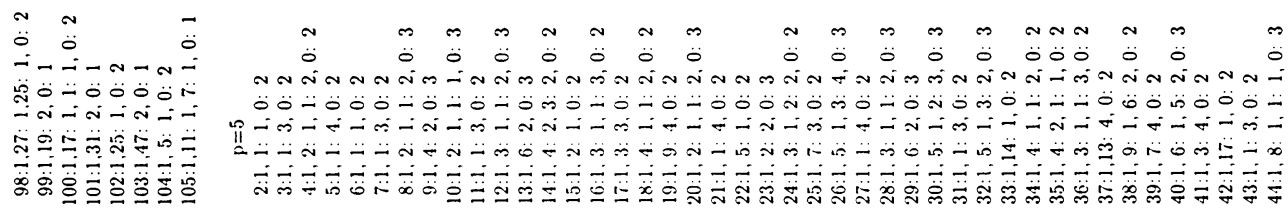

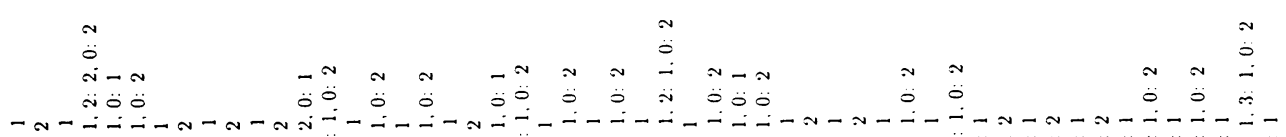
öo

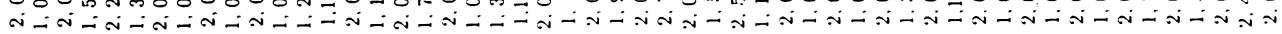

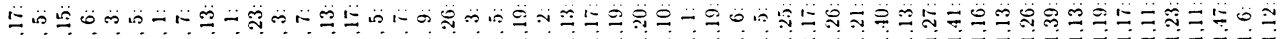

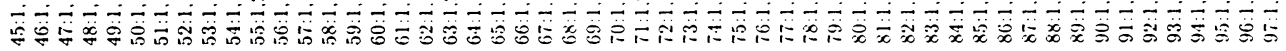

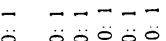

$\circ 00000$

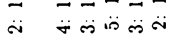

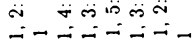

ले $\ddot{0} \ddot{0} \ddot{0} \ddot{\infty}$ iे

$\because-1=-1-1$

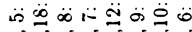

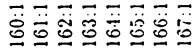

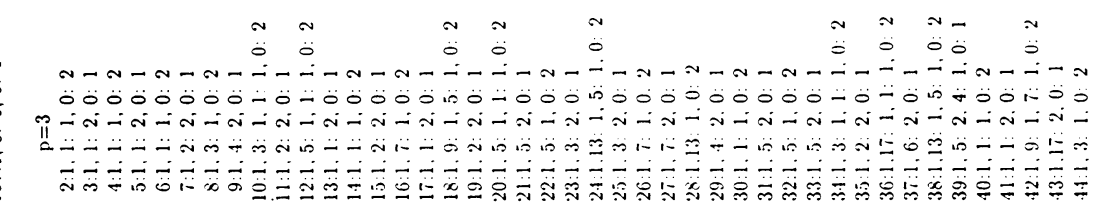


$\stackrel{\Upsilon}{\dddot{0}}$

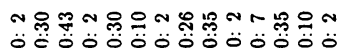

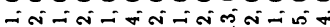

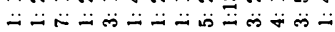
$\therefore$ 舟

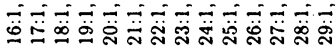

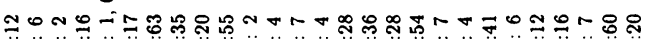

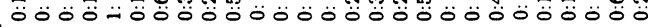

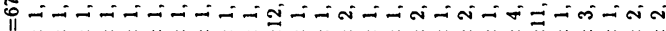

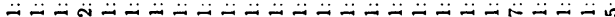

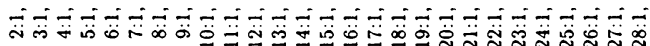

$=\infty \equiv \infty \cong r m$ 苑 $\ddot{0} \ddot{0} \ddot{0} \ddot{0} 0$

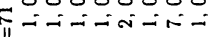

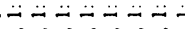

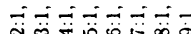

$\sin N \mathbb{m} \infty$ $\ddot{0} \ddot{0} \ddot{0} \ddot{0} \ddot{0}$

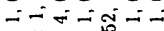

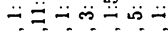
ลั

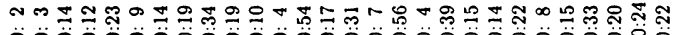

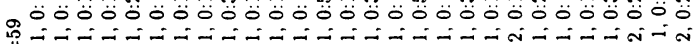
II

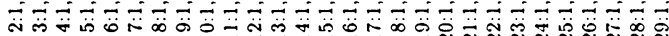

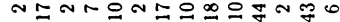

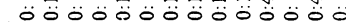

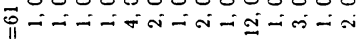
过 $\ddot{-} \ddot{-} \ddot{-} \ddot{-} \ddot{-} \ddot{-} \ddot{-}$ 的

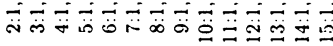

๓

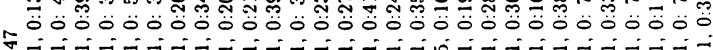
II

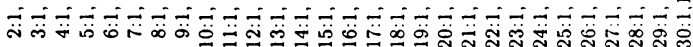

⿰幺𠃌

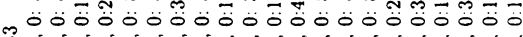
iil

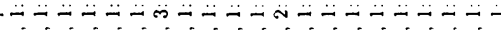

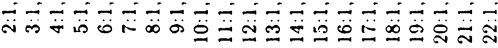

च.

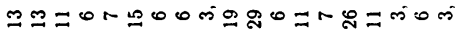

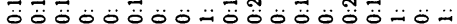
ZAo

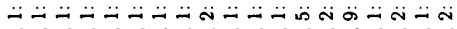

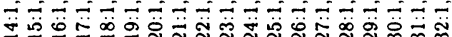

m

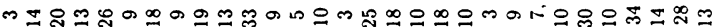

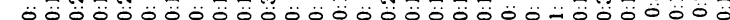

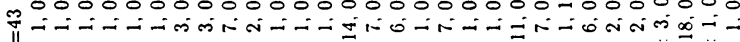
过

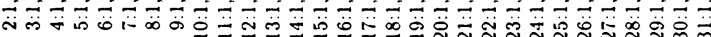

\begin{tabular}{|c|c|c|}
\hline สิ & 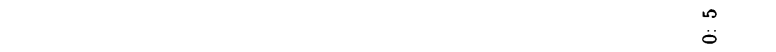 & $\stackrel{m}{\ddot{0}}$ \\
\hline 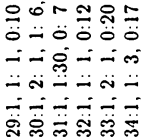 & 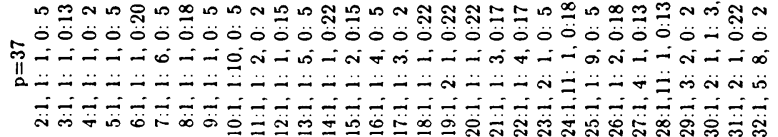 & 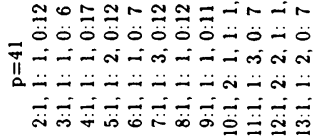 \\
\hline
\end{tabular}

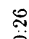

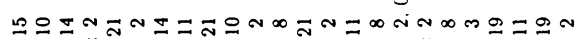

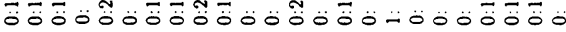

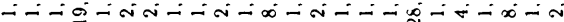

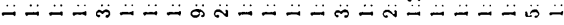

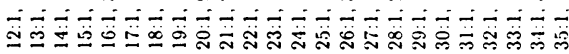

ส

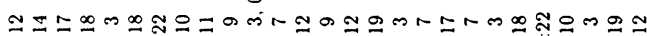
ôة

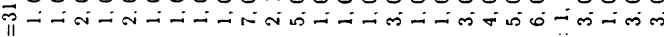
- - - - - - - -

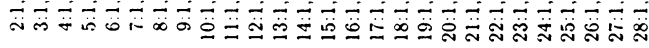

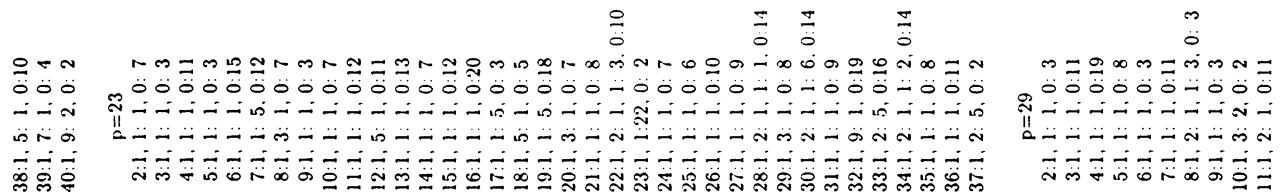

$$
\begin{array}{lll}
\infty & 0 & 0
\end{array}
$$$$
\because 00
$$

nnorrio

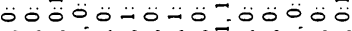

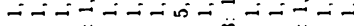

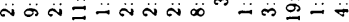

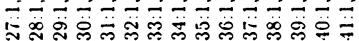

$\because 2$

$\stackrel{m}{\dddot{0}}$

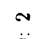

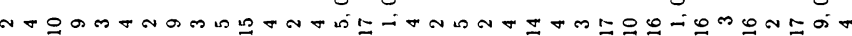

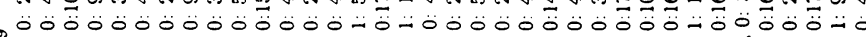

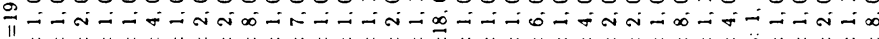

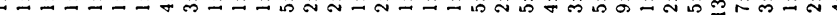

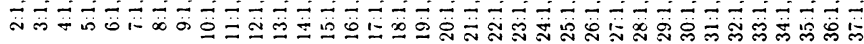




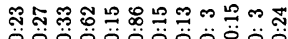

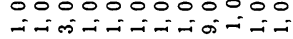

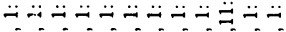

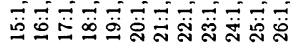

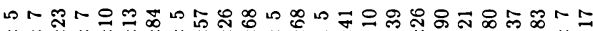

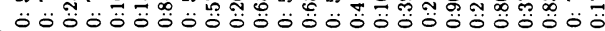

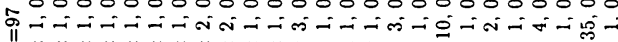

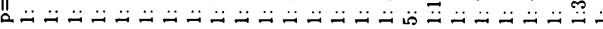

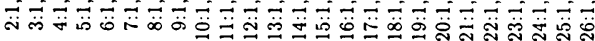

ণัษ $\stackrel{\infty}{\ddot{0}}$

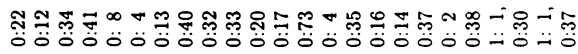

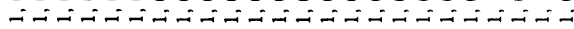

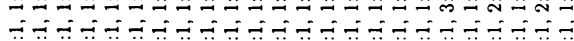

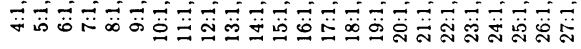

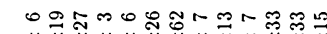

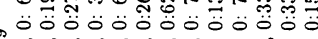

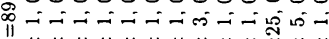
$\therefore \div \div \div \div \div=\div \div$

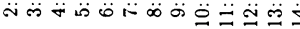

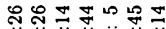

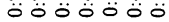
की

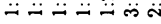

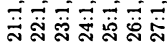

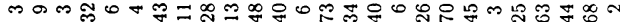

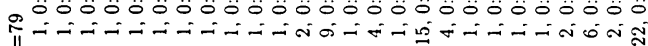

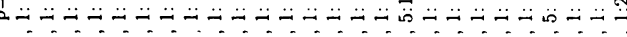

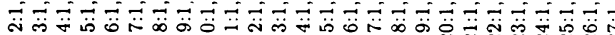

สุณ $\ddot{0} \ddot{0} \ddot{0} \ddot{0} \ddot{0} \ddot{0} \ddot{0} \ddot{0} \ddot{0} \ddot{0} \ddot{0} \ddot{0} \ddot{0} \ddot{0} \ddot{0}$

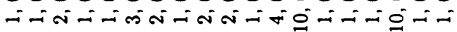

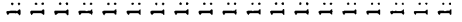

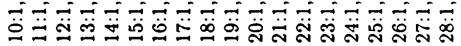

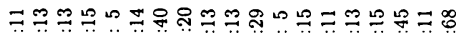

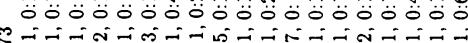

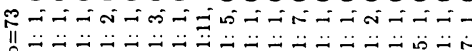

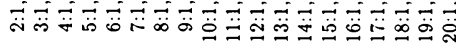

